\title{
Fórmulas de emisión y consumo de los debates televisivos en España en las elecciones legislativas de 2019
}

\section{Broadcast formulas and television debates consumption in Spain in the 2019 parliamentary election}

\author{
Ana González-Neira; Salomé Berrocal-Gonzalo; Patricia Zamora-Martínez
}

Cómo citar este artículo:

González-Neira, Ana; Berrocal-Gonzalo, Salomé; Zamora-Martínez, Patricia (2020). "Fórmulas de emisión y consumo de los debates televisivos en España en las elecciones legislativas de 2019". El profesional de la información, v. 29, n. 2, e290221.

https://doi.org/10.3145/epi.2020.mar.21

Artículo recibido el 10-10-2019 Aceptación definitiva: 02-03-2020

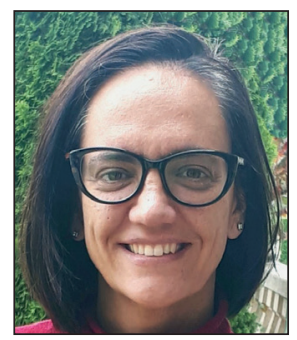

Ana González-Neira https://orcid.org/0000-0002-6369-0323

Universidade da Coruña Facultad de Comunicación

Campus de Elviña

15007 A Coruña, España

ana.gneira@udc.es

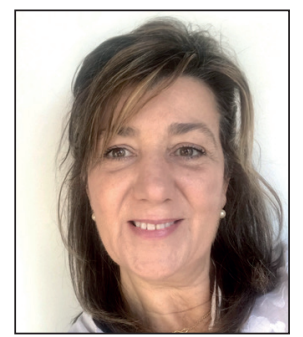

Salomé Berrocal-Gonzalo

https://orcid.org/0000-0002-0483-0509

Universidad de Valladolid

Facultad de Filosofía y Letras

Plaza del Campus, s/n.

47011 Valladolid, España

salomeb@hmca.uva.es

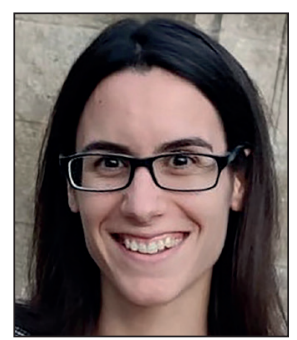

\section{Patricia Zamora-Martínez \\ Universidad de Valladolid \\ Facultad de Filosofía y Letras \\ Plaza del Campus, s/n. \\ 47011 Valladolid, España \\ patricia.zamora@uva.es}

https://orcid.org/0000-0002-5730-7295

\section{Resumen}

Los debates electorales televisivos son uno de los acontecimientos más relevantes en período electoral por su elevada audiencia y centrar la agenda mediática. Esta investigación examina la transmisión de este formato ante la multiplicación de pantallas, la difusión en redes sociales, la introducción de los servicios on demand y el consumo en movilidad junto con la aparición de nuevos actores televisivos. Este estudio de tipo cuantitativo analiza los debates emitidos en las elecciones legislativas de España, celebradas el 28 de abril de 2019, para conocer su expansión más allá del consumo tradicional televisivo y el engagement alcanzado. El método de trabajo combina la monitorización de distintas plataformas. Las conclusiones indican que la emisión de estos debates se produce a través de nuevas ventanas de distribución, originando un elevado índice de seguimiento en Twitter. Las estrategias de emisión de los grupos televisivos difieren.

\section{Palabras clave}

Debates electorales; Elecciones; Televisión; Audiencia social; Comunicación política; Medios sociales; Redes sociales; Twitter; TVE; Atresmedia; España. 


\begin{abstract}
Television electoral debates are considered one of the most relevant events during the election period due to their high audience and also because of the power they generate to direct the media agendas. The present research examines the transmission of this format in front the proliferation of screens, diffusion on the social networks, introduction of on demand services, and on the go consumption together with the emergence of new television performers. This quantitative study analyses the debates issued during the parliamentary elections in Spain, which took place on the $28^{\text {th }}$ of April 2019, in order to learn about their impact further than traditional television consumption and the engagement achieved. The methodology employed combined the monitoring of different platforms. The conclusions obtained support that the emission of this kind of debates takes place through new distribution channels, generating a high tracking rate on Twitter. The broadcasting strategies of the television chains differ.
\end{abstract}

\title{
Keywords
}

Electoral debates; Election; TV; Television; Social audience; Political communication; Prosumers; Social media; Social networks; Twitter; Atresmedia; TVE; Spain.

\section{Introducción}

El 23 de abril de 2019 tuvieron lugar las elecciones generales en España, una celebración en un contexto particular ya que fueron convocadas anticipadamente por el entonces presidente de Gobierno, Pedro Sánchez, al rechazar el Congreso de los Diputados los Presupuestos Generales de 2019.

Desde el punto de vista de la comunicación política, estos comicios se presentan especialmente interesantes al contar con nuevas plataformas de difusión junto a redes sociales consolidadas, cuya actividad se convierte en significativa en la campaña electoral.

Como había sucedido en convocatorias electorales anteriores, los debates electorales televisados fueron los grandes eventos mediáticos de la campaña, tanto por la audiencia conseguida, como por centrar la agenda mediática al producirse informaciones relacionadas con encuestas sobre el ganador/perdedor, el formato utilizado, los discursos emitidos por los líderes políticos, así como por la emisión de programas de análisis previos y posteriores a la emisión del debate, entre otras cuestiones (Rímoli, 2018; Van-Holsteyn, 2018; Pallarés-Navarro; García-Ortega, 2017; Humanes, 2014; Marín, 2003).

\subsection{Experiencia española en la emisión de debates electorales}

Televisión Española fue la encargada de organizar los dos primeros debates de la campaña de 2019. En el primero participaron representantes de los principales partidos políticos. La 1 de TVE obtuvo en esa ocasión una audiencia de 1.794.000 espectadores, mientras que, en el segundo, que contó con la presencia de los candidatos a la presidencia del gobierno, logró 7.245.000. El tercero de los debates lo organizó el Grupo Atresmedia y en él también estuvieron presentes los principales candidatos a la presidencia. Antena 3 alcanzó una audiencia media de 5.283.000 espectadores y La sexta, 4.194 .000 (Barlovento, 2019).

La retransmisión de estos debates se produce en pleno proceso de espectacularización de la política

A este escenario de consumo tradicional televisivo cabe sumar la actividad desempeñada por nuevas plataformas de emisión y expansión del formato, así como de redes sociales consolidadas, que permiten incorporar la interactividad de los usuarios. Todo ello en un panorama de mayor pluralismo político (con la incorporación de partidos como Podemos, Ciudadanos y Vox) y en un marco mediático dominado por el entretenimiento.

Desde 1993, fecha en la que se emitieron en España los dos primeros televisados, se han retransmitido ocho debates electorales con la presencia de los principales candidatos a la presidencia del Gobierno ${ }^{1}$ (tabla 1).

En esta evolución histórica de los debates electorales en España se observa el proceso de cambio político que ha experimentado el país en los últimos cinco años, ya que se ha abandonado el tradicional bipartidismo de la Transición.

“La fragmentación electoral comenzó con las elecciones al Parlamento Europeo, continuó en las elecciones autonómicas de 2015 y se consolidó en las elecciones legislativas celebradas el 20 de diciembre de este mismo año. Con Ciudadanos y Podemos cambia el sistema de partidos y cambia también la comunicación política" (Martín; Vázquez; Cebrián, 2017, p. 63).

Además, se ha producido un notable cambio en el panorama televisivo. La retransmisión de estos debates se produce en pleno proceso de espectacularización de la política. La información considerada seria como la economía y la política se presenta, en la actualidad, de manera frívola y espectacular:

"si el hecho no exhibe espectacularidad en su conjunto se busca el aspecto que lo tenga para resaltarlo" (Cebrián-Herreros, 2004, p. 21). 
Tabla 1. Debates electorales celebrados en España

\begin{tabular}{|c|c|c|c|c|}
\hline Año & Día & Promotor & Moderador & Participantes \\
\hline \multirow{2}{*}{1993} & $24 / 05$ & Antena 3 & Manuel Campo Vidal & \multirow{2}{*}{$\begin{array}{l}\text { José María Aznar } \\
\text { Felipe González }\end{array}$} \\
\hline & $31 / 05$ & Tele 5 & Luis Mariñas & \\
\hline \multirow{2}{*}{2008} & $25 / 02$ & Academia de la Televisión (20 cadenas) & Manuel Campo Vidal & \multirow{2}{*}{$\begin{array}{l}\text { Mariano Rajoy } \\
\text { José Luis Rodríguez Zapatero }\end{array}$} \\
\hline & $3 / 03$ & Academia de la Televisión (22 cadenas) & Olga Viza & \\
\hline 2011 & $7 / 11$ & Academia de la Televisión (17 cadenas) & Manuel Campo Vidal & $\begin{array}{l}\text { Mariano Rajoy } \\
\text { Alfredo Pérez Rubalcaba }\end{array}$ \\
\hline \multirow[t]{2}{*}{2015} & $7 / 12$ & $\begin{array}{l}\text { Antena } 3 \\
\text { La sexta }\end{array}$ & $\begin{array}{l}\text { Ana Pastor } \\
\text { Vicente Vallés }\end{array}$ & $\begin{array}{l}\text { Pablo Iglesias } \\
\text { Albert Rivera } \\
\text { Soraya Saénz de Santamaría } \\
\text { Pedro Sánchez }\end{array}$ \\
\hline & $14 / 12$ & Academia de la Televisión (12 cadenas) & Manuel Campo Vidal & $\begin{array}{l}\text { Mariano Rajoy } \\
\text { Pedro Sánchez }\end{array}$ \\
\hline 2016 & $13 / 06$ & Academia de la Televisión (17 cadenas) & $\begin{array}{l}\text { Ana Blanco } \\
\text { Pedro Piqueras Vicente Vallés }\end{array}$ & $\begin{array}{l}\text { Pablo Iglesias } \\
\text { Albert Rivera } \\
\text { Pedro Sánchez } \\
\text { Mariano Rajoy }\end{array}$ \\
\hline \multirow{2}{*}{2019} & $22 / 04$ & $\begin{array}{l}\text { RTVE } \\
\text { (11 cadenas) }\end{array}$ & Xabier Fortes & $\begin{array}{l}\text { Pablo Casado } \\
\text { Pablo Iglesias } \\
\text { Albert Rivera } \\
\text { Pedro Sánchez }\end{array}$ \\
\hline & $23 / 04$ & $\begin{array}{l}\text { Atresmedia } \\
\text { (Antena 3; La sexta) }\end{array}$ & $\begin{array}{l}\text { Ana Pastor } \\
\text { Vicente Vallés }\end{array}$ & $\begin{array}{l}\text { Pablo Casado } \\
\text { Pablo Iglesias } \\
\text { Albert Rivera } \\
\text { Pedro Sánchez }\end{array}$ \\
\hline
\end{tabular}

Una situación que se manifiesta en la aparición de programas en donde se difuminan las líneas entre información y entretenimiento (Martín; Vázquez; Cebrián, 2017, p. 53). La actual parrilla televisiva evidencia la aparición de un nutrido número de programas que

"o bien transforman la política en un espectáculo al incluirla en un formato de show, o bien la reflejan en programas de parodia televisiva" (Berrocal-Gonzalo; Cebrián-Guinovart, 2009, p. 42).

Un panorama que afecta a los partidos políticos, ya que los espectadores acuden a estos espacios para profundizar en aspectos de la vida privada de los protagonistas, transformados en celebrities (Ortells-Badenes, 2009, p. 343) y que, también, influye en el desarrollo de los debates electorales en los últimos años, al triunfar las formas, las frases en clave espectacular o lo anecdótico frente a los argumentos racionales y las propuestas de gobierno.

\subsection{Expansión del consumo televisivo en la audiencia social y engagement}

La lucha por mantener el liderato frente a internet ha cambiado notablemente las prácticas de consumo televisivo. Éste se ha diversificado debido a la expansión de los dispositivos móviles, la mejora en las condiciones de conexión, así como la aparición de nuevas plataformas, redes sociales y actores emisores. Todo este conglomerado ha dado lugar a la televisión líquida (Quintas-Froufe; González-Neira, 2016) en la que resulta difícil establecer los límites entre las figuras de receptores y emisores así como los parámetros relativos al momento y lugar del consumo. El consumo atawad (anytime, anywhere, any device), favorecido por los avances tecnológicos que se han comentado altera la naturaleza tradicional de este medio. Además, los habituales emisores, las cadenas de televisión, comparten esa función con actores de origen diverso (Amazon, HBO, Netflix) que conforman las OTT (over-the-top), así como con ciudadanos individuales convertidos en productores de contenido audiovisual (influencers) (De-Aguilera-Moyano; Castro-Higueras; Pérez-Rufí, 2018) que aprovechan las potencialidades de la Red, los nuevos dispositivos móviles y la consolidación de las redes sociales para compartir aquello que crean.

Frente al público tradicionalmente pasivo, consumidor únicamente de contenidos debido a la falta de medios de participación, en este nuevo ecosistema mediático se ha dado paso a una audiencia activa que no sólo presta atención a los temas emitidos por el medio televisivo, sino que a su vez participa en el nuevo entorno a través de comentarios y contenidos diversos relacionados con aquello que están viendo a través de sus pantallas.

"El modelo tradicional de la comunicación unidireccional en el que se basa el medio televisivo está dando paso a un modelo participativo, caracterizado por el papel activo de la audiencia en los programas y su creciente interacción con los contenidos, que se ve favorecido por el potencial de las nuevas tecnologías" (García-Avilés, 2011, p. 190).

Esta reciente cultura participativa del público promueve el nacimiento de la denominada audiencia social, entendida como

"aquella que utiliza las redes sociales al mismo tiempo que contempla la televisión, se comporta de modo creativo y plenamente interactivo" (Congosto et al., 2013, p. 57). 
La centralidad del espectador y su papel decisivo como actor en el nuevo mundo comunicativo ha derivado en la existencia de un público que se apoya en las plataformas, medios digitales y en internet para establecer nuevas maneras de engagement (palabra que ha sido traducida en la mayoría de los casos en español como implicación, participación o interacción) con los contenidos que utilizan y consumen. Según Ballesteros-Herencia el engagement consistiría en un "proceso bidireccional" y, añade que por parte de los individuos

"haría referencia tanto a un estado cognitivo como a su manifestación, ya sea esta en forma de representaciones simbólicas efectuadas mediante utilidades de las redes sociales ("me gusta", compartir, comentar), como de gestos corporales, actitudes o expresión de opiniones” (Ballesteros-Herencia, 2019, p. 226).

En este sentido, según algunos estudios, las redes sociales serían el mejor espacio para verter contenido con claros vínculos afectivos que ayuden a potenciar la participación de los cibernautas (Papacharissi, 2015; Van-Dijck, 2013).

A su vez, esta nueva audiencia ha convertido en algo cotidiano y habitual el uso de la segunda pantalla como se puede extraer del barómetro del Centro de Investigaciones Sociológicas (CIS, 2018). Los dispositivos más usados por los ciudadanos para acceder a la Red son los teléfonos móviles (92,8\%), seguido por los ordenadores portátiles (51,8\%) y el ordenador de sobremesa (43,3\%). Además, las actividades más frecuentes que realizan en las redes sociales según el estudio de $I A B$ (2019) son: chatear/enviar mensajes (privados/públicos) a los contactos (65\%); ver vídeos, música (57\%); publicar/colgar contenidos (fotos, vídeos, noticias, música, etc.) (34\%); seguir cuentas (medios de comunicación, partidos políticos, etc.) y comentar la actualidad (31\%), rol íntimamente ligado con la participación de la audiencia social en el nuevo ecosistema mediático.

Ante esta nueva realidad, los emisores tradicionales de televisión están redefiniendo su estrategia de difusión (Boyle, 2019) y de acercamiento hacia los públicos más jóvenes que están abandonando el consumo de este tipo de canales.

Uno de los últimos episodios en la televisión es el Social media live television (smltv), derivado del Social media live streaming, también conocido por sus siglas de smls, que incluye la interactividad y la movilidad como valores agregados principales. Como indica Apablaza-Campos (2018b) se trata de una simbiosis entre una especie tradicional como la televisión y otra de las nuevas, las redes sociales.

En este contexto mediático las audiencias se han dotado de mayores posibilidades de interacción con los contenidos televisivos gracias a los avances tecnológicos y a la cultura de la participación (Jenkins; Ito; Boyd, 2015). Las redes sociales, a través de la televisión social (González-Neira; Quintas-Froufe, 2015), han posibilitado nuevos canales de comunicación, así como la existencia de un feedback entre los antiguos roles de emisor y receptor (si bien algunas investigaciones han demostrado que esta posibilidad no está totalmente explotada: Bergillos, 2017) que a su vez mejoran la fidelización del televidente. El espectador de la televisión actual posee un perfil activo, multimedia, multiplataforma, en contacto constante con la Red y con las tecnologías de la información y acostumbrado al visionado online (García-García; Vinader-Segura; Abuín-Vences, 2012). Por lo tanto, ante estas prácticas de consumo deben satisfacer una experiencia de usuario cada vez más exigente.

Estos factores alteran el escenario mediático con repercusiones directas en el sistema político español. De ahí el estudio que aquí se presenta.

\section{Objetivos y metodología}

Esta investigación contribuye a explorar las extensiones que tiene la televisión más allá de su canal de distribución tradicional. A través de un hecho noticioso significativo como un debate electoral, se estudian las posibilidades que las cadenas españolas están aplicando en su estrategia actual. Se parte de la hipótesis de que la apertura a nuevas ventanas de difusión en contenidos aumenta enormemente las posibilidades de engagement porque supone aprovechar los mecanismos que cada una de esas redes sociales y plataformas proporcionan.

En esta investigación se estudia de qué modo la emisión de los debates electorales de las elecciones legislativas de abril de 2019 se ha adaptado al novedoso panorama mediático. Las preguntas de investigación son las siguientes:

- ¿Cuál ha sido la estrategia de los grupos de comunicación en la emisión de los debates?

- ¿Las cadenas emisoras han aprovechado las nuevas ventanas de distribución de contenido audiovisual en la difusión de este formato?

- ¿Qué resultados han tenido con su táctica de transmisión?

- ¿Cómo ha sido la participación de la audiencia social a través de las distintas plataformas durante la retransmisión de los debates? 
El estudio busca comprobar si los canales propietarios de la señal televisiva favorecieron la difusión de este formato en campaña electoral. Asimismo, se presta atención a las posibilidades de participación, entendida como la que se produce mediante la publicación de tweets o comentarios sobre lo que se está viendo, y al aprovechamiento que han hecho de las nuevas fórmulas comunicativas, como manifestación del engagement. El análisis, de tipo cuantitativo, examina las oportunidades que brindan las nuevas plataformas como difusoras de contenido en directo.

El objeto de estudio se refiere a los debates electorales emitidos por las cadenas generalistas españolas en el territorio nacional durante la campaña de abril de 2019. A diferencia de otras ocasiones estos debates no fueron promovidos por la Academia de la Televisión, como gran parte de los celebrados hasta 2019. Este hecho altera notablemente la difusión de este espacio ya que la iniciativa de la emisión pasa en exclusiva a un grupo mediático. De ahí el interés de esta investigación en dilucidar qué tipo de estrategia se implementó ante este contenido tan sensible y si ésta aprovechó las nuevas ventanas de difusión.

En una primera fase de trabajo se realizó una revisión documental relacionada con el objeto de estudio y posteriormente se monitorizó la emisión de los debates electorales en varias plataformas, concretamente: Twitter, Facebook, Periscope, YouTube, webs y apps. Para ello se recogieron datos relativos al visionado y número de comentarios a estos espacios en función de la naturaleza de cada plataforma analizada: Twitter, Facebook, Periscope, YouTube. Asimismo, se comprobó su posible emisión en las webs corporativas y las apps propias. Se monitorizaron las cuentas oficiales de los canales y grupos mediáticos que emitieron estos espacios. Dado que a través de los sistemas smltv (Apablaza-Campos, 2018a; 2018b; Apablaza-Campos; Codina 2018) se combina el visionado en directo con la emisión de comentarios suministrados por cada plataforma observada, la investigación también aborda este tipo de producción de los espectadores. Concretamente, se han examinado los comentarios vertidos en Twitter por ser la red social en la que tradicionalmente se produce el mayor intercambio de opiniones en abierto sobre contenidos televisados en directo y que permite una mejor monitorización (Gómez-Domínguez, 2019).

La aplicación utilizada para el análisis de los tweets es la versión online de pago Twitonomy. Para una mayor fiabilidad del estudio, la descarga realizada desde la plataforma de monitorización ha sido efectuada en dos ocasiones para recuperar el mayor volumen de mensajes de la audiencia social durante la retransmisión de los dos encuentros (quedan excluidos de la muestra todos aquellos mensajes publicados una vez finalizada la retransmisión). Twitonomy realizó el seguimiento de los comentarios publicados en Twitter a través de los dos hashtags oficiales que se crearon para ambos encuentros: \#DebateAtresmedia y \#ELDEBATEenRTVE".

La investigación se enmarca en la teoría de las audiencias activas (Livingstone, 2004) en los estudios referidos a debates electorales (Casado-Ruiz, 2012; Herranz-Rubio, 2020), participación de la audiencia en dichos eventos televisados (Saavedra-Llamas; Rodríguez-Fernández, 2018; Lee; Choi, 2018; Márquez-Martínez, 2017; Kalsnes; Krumsvik; Storsul, 2014; Elmer, 2013), así como las oportunidades que proporcionan las redes sociales para su emisión en directo (Herrero-de-la-Fuente; García-Domínguez, 2019; Apablaza-Campos, 2018a; 2018b; 2017; Apablaza-Campos; Codina, 2018; Raman; Tyson; Sastry, 2018; Herrero-de-la-Fuente, 2017; Marta-Lazo; Mancho-Laiglesia; Villa-Navarro, 2017; Mancebo-García, 2016) y la respuesta de la audiencia social tanto a nivel internacional (D’heer; Verdegem, 2015; Larsson, 2013; Highfield; Harrington; Bruns, 2013;) como nacional (Gómez-Domínguez, 2019; Halpern; Quintas-Froufe; Fernández-Medina, 2016; Saavedra-Llamas; Rodríguez-Fernández, 2016; Gallardo-Camacho; Lavín; Fernández-García, 2016; Claes; Deltell; Congosto, 2015; Claes; Deltell, 2015; Rodríguez-Mateos; Hernández-Pérez, 2015; Gómez-Rubio; López-Vidales, 2015; Gómez-Aguilar; Paniagua-Rojano; Farias-Batlle, 2015; Quintas-Froufe; González-Neira; Díaz-González, 2015; García-Ferrando, 2014; Deltell; Claes; Osteso, 2013; Congosto et al., 2013).

En cuanto a la conexión del estudio con otros trabajos anteriores que vincule las nuevas formas de distribución de contenido audiovisual con la emisión de debates electorales, cabe señalar que no se ha localizado ninguna referencia bibliográfica, lo que hace que esta investigación sea muy pertinente para avanzar en este ámbito.

Por lo que se refiere a la muestra examinada, se realizó la monitorización de las plataformas en los tres debates electorales realizados en abril de 2019, mientras que el estudio particular en Twitter se centró en el examen de los dos debates que contaron con los candidatos a la presidencia del Gobierno.

Del debate transmitido por Televisión Española el 22 de abril se recuperaron 6.111 comentarios, 3.045 se publicaron durante la primera hora (22:00 a 23:00) y 3.066 en la segunda hora (23:00 a 23:30). En el caso del emitido por Atresmedia el 23 de abril se descargó un total de 6.064 mensajes, de los cuales 3.055 fueron publicados durante la primera hora del evento (22:00 a 23:00) y 3.009 fueron enviados en la segunda parte (23:00 a 00:20).

De ambos debates, atendiendo a las franjas horarias citadas, Twitonomy proporciona métricas de participación (retweets y favoritos) de los mensajes emitidos por los usuarios. De este modo, la investigación puede inferir resultados vinculados al objetivo acerca del engagement logrado por ambas cadenas a través de las segundas pantallas y, en concreto, a través de la red social Twitter. 


\section{Resultados}

Tras la monitorización de los debates de abril de 2019 se presentan los datos tanto de las ventanas de difusión de los mismos, como de su respectivo seguimiento. Asimismo, se aportan datos sobre la participación de la audiencia a través de la producción de mensajes en las diversas plataformas.

\subsection{Ventanas de difusión}

\section{Debate del 16 de abril de 2019}

Fue retransmitido en directo por $L a 1$ y el canal $24 \mathrm{H}$ de Televisión Española, así como por la cuenta de RTVE en YouTube, la web de RTVE, Periscope (cuenta de $24 H$ ), las aplicaciones móviles +24 de TVE y de RNE. Además, en Facebook, un post publicado en la cuenta de RTVE reenviaba a todas las ventanas de difusión anteriores. La audiencia tradicional fue de 1.794 .000 espectadores en La 1 con un $11,8 \%$ de cuota de pantalla. Durante esa noche fue el programa más visto en su franja horaria.

En Twitter, desde las cuentas de @24h_TVE y @La1_TVE se retransmitió en directo y a pesar de llegar a ser trending topic, no consta como una de las cinco emisiones con mayor éxito en audiencia social de esa semana (15-21 de abril).

En YouTube 2.841 usuarios se conectaron simultáneamente y hubo más de 51.000 visualizaciones en las primeras 24 horas del espacio. Hasta principios de octubre de 2019, esa cifra se había incrementado hasta 275.350 visualizaciones que habían producido 2.521 comentarios.

La plataforma Periscope alcanzó 38.177 espectadores totales, de los cuales 5.630 fueron en repetición. El tiempo medio de visualización fue de 2 minutos y 8 segundos, un consumo muy breve.

Tras este análisis se comprueba que RTVE amplió sus tradicionales ventanas de visionado y abrió la difusión a través de nuevas experiencias en plataformas. También se constata que el afán de servicio público dirige la estrategia de RTVE en sus emisiones. Algunos medios escritos como El país y El mundo transmitieron en sus respectivas webs la señal en directo de RTVE.

\section{Debate del 22 de abril de 2019}

El debate promovido por RTVE fue retransmitido el 22 de abril por $L a 1$ y el canal $24 \mathrm{H}$, asimismo fue emitido en simultáneo en Castilla La Mancha Media, Canal Extremadura, Canal Sur, Trece TV, Telemadrid, IB3 y Aragón TV. Esta emisión general tuvo una audiencia total de 8.886.000 espectadores y un share del $43,8 \%$. La mayor parte de la audiencia se concentró en La 1 con 7.245.000 espectadores y una cuota de $35,7 \%$. La web de la corporación pública también se sumó a la retransmisión en directo, así como la app de Noticias de RTVE.

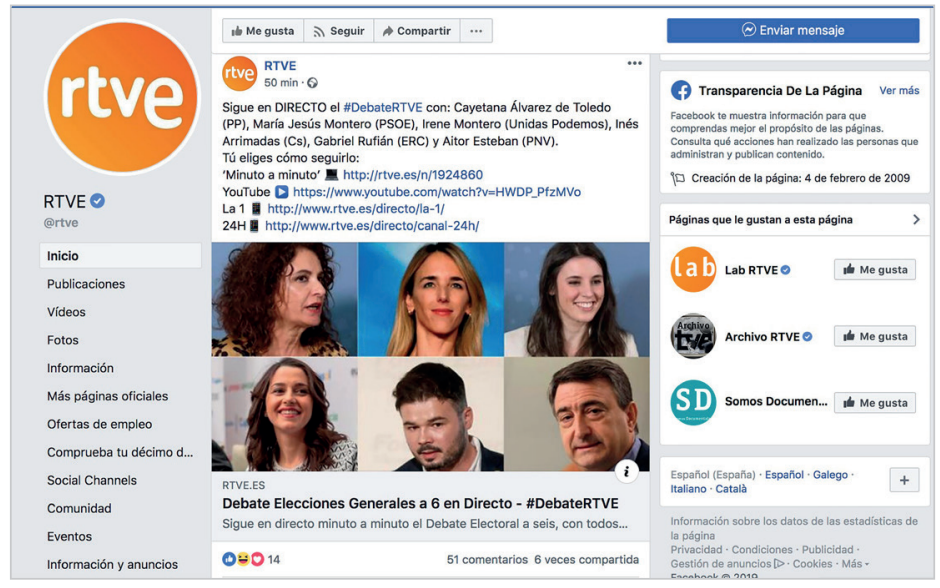

Imagen 1. Post publicado en la cuenta de RTVE en Facebook

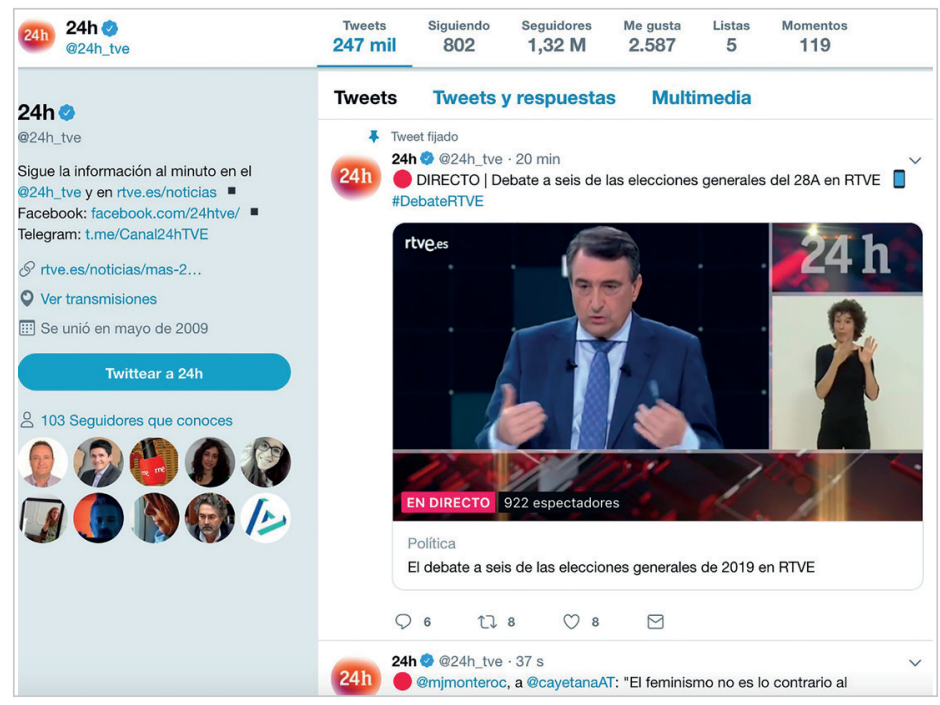

Imagen 2. Retransmisión en Twitter desde la cuenta @24h_TVE

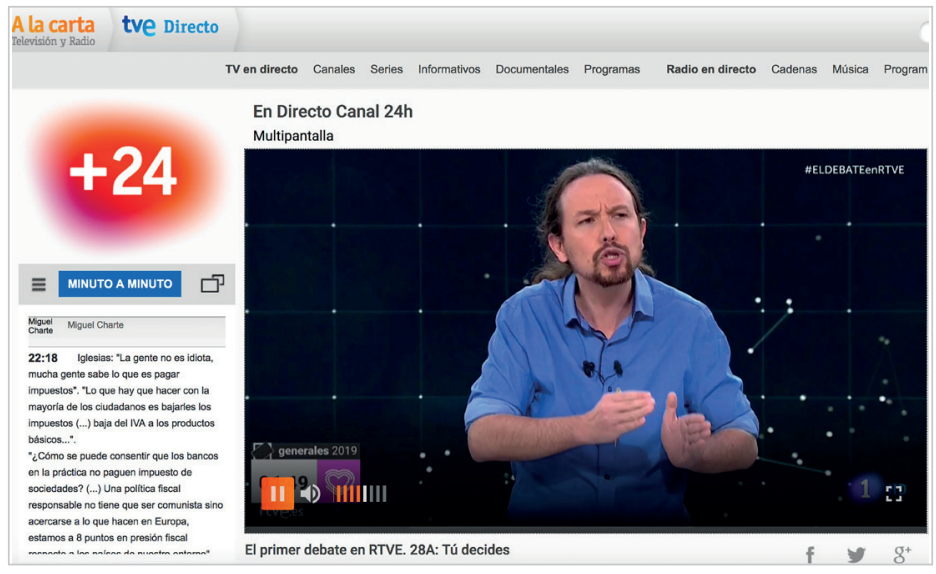

Imagen 3. Emisión del debate a través de la web de RTVE

Al igual que en el caso anterior se transmitió en nuevas plataformas y redes sociales. En Twitter las cuentas de @24h_ TVE y @La1_TVE lo emitieron en directo. En esta red social se produjeron 147.900 .000 impresiones y 773.400 tweets, un volumen muy elevado en los índices habituales de audiencia social. 
A diferencia del anterior debate, hubo una emisión en directo en Facebook Live que alcanzó un elevado número de comentarios. En los primeros 14 minutos, 493 personas la veían en directo y habían participado con 695 comentarios, sin duda la más alta actividad en proporción espectadores/comentarios de todas las plataformas examinadas. Esta propensión a la participación se mantuvo hasta el final del debate, ya que la primera cifra casi se había duplicado, mientras que la segunda se había multiplicado por cinco al llegar a los 3.743 comentarios.

Por lo que se refiere a YouTube, esta retransmisión a través de la cuenta de RTVE superó los 13.800 espectadores en directo. Además, 12 horas después del inicio de su emisión, consiguió 93.000 visualizaciones y 485 comentarios. A principios de octubre de 2019 se habían alcanzado las 549.679 visualizaciones y los 3.281 comentarios.

Al comparar los datos de estas dos últimas plataformas se deduce que YouTube figura principalmente como una red de visionado y menos de participación, mientras que en Facebook la intervención es mucho mayor y se manifiesta en un número superior de comentarios. Los espectadores respetan la naturaleza inicial de cada una de estas plataformas, de ahí que se produzca una preferencia por comentar en Facebook mientras que el visionado es mayor en YouTube.

RTVE también distribuyó la señal a otros medios como los diarios El mundo y El país, tradicionalmente alejados de la emisión de contenidos audiovisuales en directo.

La cuenta de RTVE en Periscope también fue otra ventana de difusión de los debates electorales. Esta plataforma contó con 227.829 espectadores en directo, de los cuales 63.189 fueron en repetición. Del mismo modo que en el caso anterior, el tiempo medio de visualización por espectador fue muy breve, de dos minutos y cuatro segundos.

Al igual que en el debate anterior, RTVE mantuvo la misma estrategia de integración del directo en plataformas y cedió la señal a múltiples emisores.

\section{Debate de 23 de abril de 2019}

El debate fue retransmitido en exclusiva por las dos cadenas principales del grupo Atresmedia así como Antena 3 Internacional, que no cedieron la señal a ningún tercero. Antena 3 alcanzó una audiencia media de 5.283.000 espectadores y un share de $27,2 \%$ frente a los 4.194 .000 espectadores de La sexta que supusieron un $21,6 \%$.

La emisión no tuvo ninguna ventana de difusión extra en Facebook, Twitter, Periscope o YouTube, y tampoco en los principales diarios escritos.

En YouTube fue una cuenta particular (Carlos 91391) la que parcialmente transmitió el debate con más de cinco mil espectadores en directo. En la web, el debate también fue difundido a través de Atresplayer para sus suscriptores.

Tabla 2. Presencia de ventanas de visionado

\begin{tabular}{|l|c|c|}
\cline { 2 - 3 } \multicolumn{1}{c|}{} & \multicolumn{1}{c|}{$\begin{array}{c}\text { Debate } \\
\mathbf{1 6 / 0 4 / 2 0 1 9}\end{array}$} & $\begin{array}{c}\text { Debate } \\
\mathbf{2 2 / 0 4 / 2 0 1 9}\end{array}$ \\
\hline $\begin{array}{l}\text { Emisión lineal (TVE, 24H, TVE Internacional, Antena 3, La sexta, Antena 3 Internacional, } \\
\text { otros en simulcast) }\end{array}$ & $\mathrm{X}$ & $\mathrm{X}$ \\
\hline Twitter & $\mathrm{X}$ & $\mathrm{X}$ \\
\hline Facebook & $\mathrm{X}$ & $\mathrm{X}$ \\
\hline Periscope & $\mathrm{X}$ & $\mathrm{X}$ \\
\hline YouTube & $\mathrm{X}$ & $\mathrm{X}$ \\
\hline Web corporativa & $\mathrm{X}$ & $\mathrm{X}$ \\
\hline Apps propias & $\mathrm{X}$ & $\mathrm{X}$ \\
\hline
\end{tabular}

* Sólo para suscriptores

La estrategia llevada a cabo por este grupo fue totalmente opuesta a la implementada por RTVE el día anterior, con un carácter más centralizado y carente de sinergias con nuevas plataformas y redes sociales que favorecieran la participación y el seguimiento. 
Tabla 3. Audiencia lineal y audiencia social de los debates electorales

\begin{tabular}{|l|l|c|c|c|}
\cline { 3 - 5 } \multicolumn{2}{c|}{} & \multicolumn{2}{c|}{ Audiencia lineal } & \multicolumn{2}{c|}{ Audiencia social } \\
\cline { 2 - 5 } \multicolumn{2}{c|}{} & Total de espectadores & Cuota de pantalla & Impresiones \\
\hline \multirow{2}{*}{ Debate TVE } & 16 de abril & 1.794 .000 & $11,8 \%$ & - \\
\cline { 2 - 5 } & 22 de abril & 8.886 .000 & $43,8 \%$ & 147.900 .000 \\
\hline Debate de Atresmedia & 23 de abril & 9.477 .000 & $48,7 \%$ & 210.300 .000 \\
\hline
\end{tabular}

\subsection{Audiencia social}

Tras la monitorización realizada se concluye que el mayor índice de participación, entendida en ese caso como producción de mensajes, tiene lugar en la red social Twitter a través de la denominada audiencia social. Según los datos facilitados por Kantar Media, la audiencia social fue superior en el debate retransmitido por Atresmedia (210.300.000 impresiones, frente a las 147.900.000 de RTVE). Esta superioridad también se percibe en el número de tweets que ambos espacios provocaron, ya que el debate de Atresmedia produjo 1.400 .000 tweets frente a los 773.400 de RTVE, según Kantar Media².

No obstante, a la hora de comparar estos datos cabe tener en cuenta que la duración del debate de Atresmedia fue superior en cincuenta minutos, por lo que hubo más tiempo para favorecer esa producción. En ambos casos estas emisiones llegaron a ser trending topic las noches de su difusión en directo y los dos espacios con mayor audiencia social del mes de abril.

Si se amplía la mirada hacia los espacios de análisis político emitidos en la programación posterior al debate se comprueba que el liderazgo del grupo mediático privado no se mantiene. El espacio Tú decides que retransmitió La 1 de TVE llegó a tener 69.600.000 impresiones y 697.800 tweets frente a los especiales de Antena 3 (El debate Atresmedia: continuación) y La sexta (Al rojo vivo: edición especial. El debate decisivo), cuya suma no alcanza los datos obtenidos por la cadena pública ya que el primero consiguió 28.700 .000 impresiones y 113.400 tweets y el segundo 18.400 .000 impactos y 79.000 tweets. En esta ocasión se produce también un factor temporal que puede desvirtuar la comparativa, frente a una duración semejante entre el espacio de Antena 3 y el de La 1, los programas especiales de comentarios y análisis político de las cadenas privadas comenzaron más tarde (00:18h.) que el de La 1 (a las 23:27h.).

El triunfo de estas retransmisiones en audiencia social se debe a que aúnan los factores que habitualmente resultan exitosos en Twitter: la emisión en directo y la controversia o el debate, una situación que también se manifiesta en la emisión de realities, competiciones deportivas (González-Neira; Quintas-Froufe, 2014), así como los contenidos que apelan a la emoción o al fandom, manteniéndose así la misma tendencia que se observaba en el consumo televisivo respecto a la espectacularización informativa y a la presencia del ingrediente entretenimiento en noticias serias (hard news).

Los internautas que tweetearon con el hashtag del evento \#DebateAtresmedia (de La sexta y Antena 3) prestaron particular atención a las intervenciones de los políticos. Así, el mensaje que más favoritos (27) y retweets (22) obtuvo durante la retransmisión de la primera hora del encuentro (22:00 a 23:00 horas) hace referencia a la intervención de Pablo Iglesias que se muestra en la imagen 5.
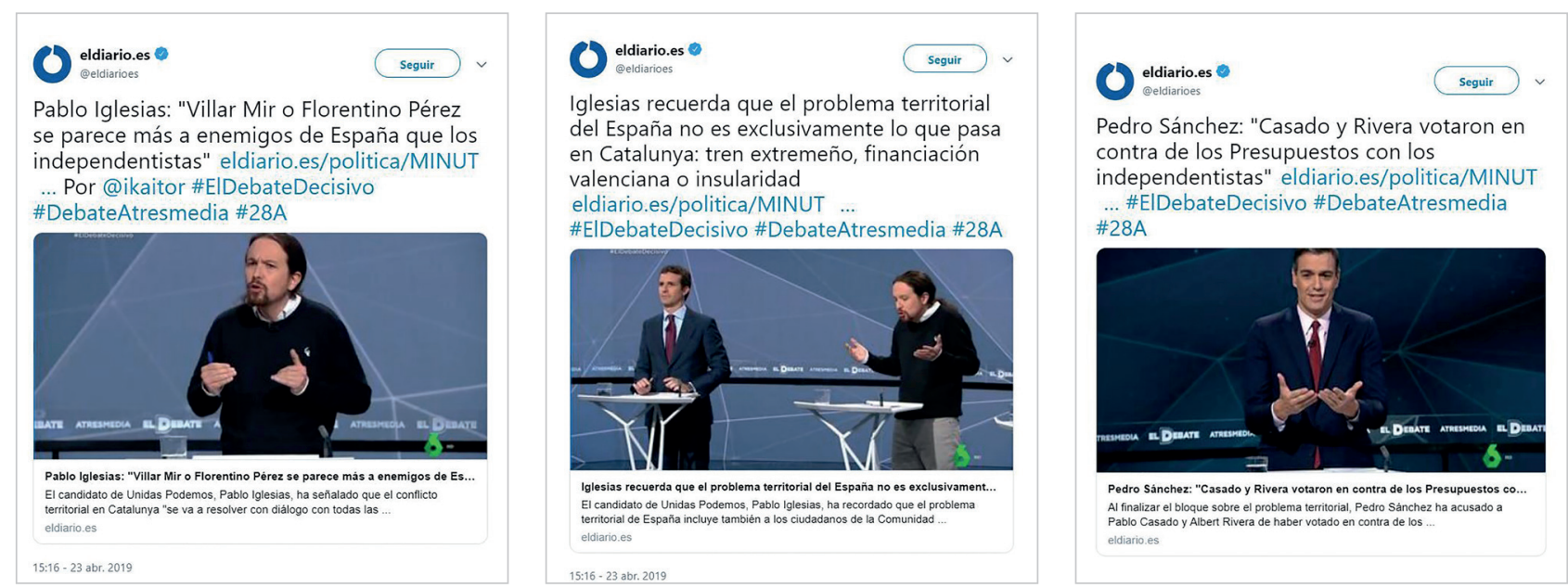

Imagen 6. Tweets con las intervenciones de los políticos 
En la segunda hora del debate (23:00 a 00:20 horas) se mantiene la tendencia señalada anteriormente y los mensajes de la audiencia social se centran en las intervenciones de los políticos. Los mensajes que más favoritos y retweets obtuvieron fueron dos intervenciones del candidato Pablo Iglesias (la primera obtuvo 66 retweets y 122 favoritos y la segunda 37 retweets y 82 favoritos), y una intervención del candidato Pedro Sánchez (17 retweets y 17 favoritos) (imagen 6).

Por otro lado, los prosumidores que tweetearon durante la primera hora (22:00 a 23:00) de emisión del debate de La 1 de TVE a través del hashtag creado para la ocasión, \#ELDEBATEenRTVE, tuvieron muy en cuenta los discursos de los candidatos, así como el desarrollo del evento. Los mensajes que más retweets y favoritos tuvieron hacen referencia a una de las intervenciones de Pablo Casado donde señala que la unidad de España está en riesgo con Pedro Sánchez (32 retweets y 21 favoritos) y otro referido al receso publicitario donde se podía ver a las empleadas de la limpieza pasando la mopa por el suelo del plató de televisión (19 retweets y 14 favoritos) (imagen 7).

En la segunda hora de debate (23:00 a 23:40) los espectadores se centraron en comentar los minutos reservados a la petición de votos de los candidatos a presidente siendo los más retweeteados uno de Pedro Sánchez (16 retweets y 25 favoritos) y otro que alude al minuto final de Albert Rivera en clave de humor (10 retweets y 11 favoritos) (imagen 8 ).

La audiencia social más activa durante la retransmisión del debate se corresponde con cuentas de medios
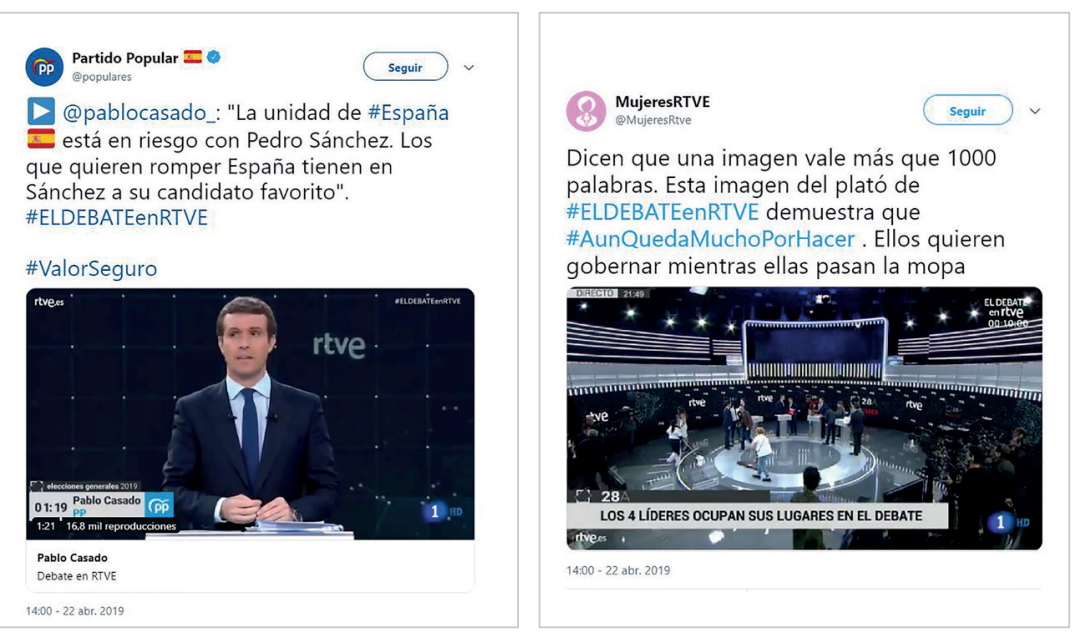

Imagen 7. Los dos mensajes con más retweets y favoritos

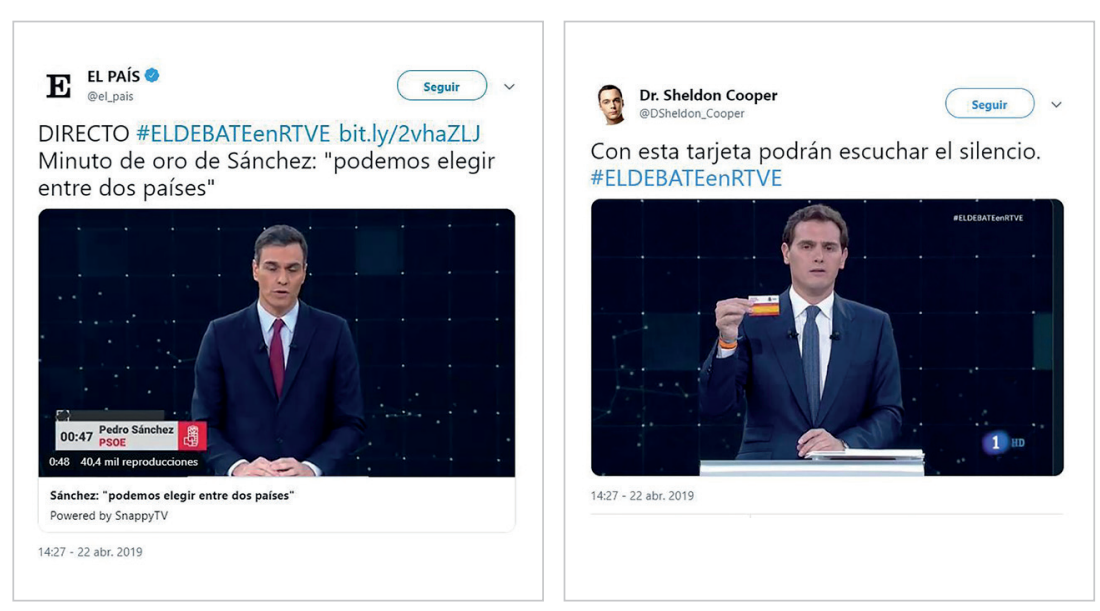

Imagen 8. Tweets que recogen la petición de voto de los candidatos de comunicación (El país, Eldiario.

es), partidos políticos (Podemos) y usuarios anónimos con un perfil falso (Dr. Sheldon Cooper). Hubo escasa interacción horizontal usuario-usuario. La interactividad de los internautas se circunscribe a unos pocos "likes" o "me gusta" dando lugar a un escenario de micronarrativas e hiperfragmentación del discurso público a través de la red social Twitter.

\section{Conclusiones}

Tras la investigación realizada se responde a las preguntas planteadas inicialmente acerca de la emisión en directo en nuevos canales de difusión. En primer lugar, se constata que se produce una notable diferencia entre la estrategia seguida por RTVE y la de Atresmedia. La primera, siguiendo las directrices del Mandato-marco a la Corporación RTVE (España, 2007), manifiesta un claro compromiso de servicio público facilitando la cesión de la señal a múltiples medios que lo solicitaron y abriendo nuevas ventanas de distribución de la señal en directo en plataformas como Twitter, Facebook, Periscope, YouTube, la web corporativa o las apps, por lo que se deduce un acercamiento hacia los retos que el escenario televisivo presenta con nuevas formas de visionado.

Por el contrario, la estrategia de Atresmedia fue centralista y no cedió la señal a ningún tercero. Además, excepto la web y la app propia, tampoco se produjo la integración del visionado en directo en plataformas y redes sociales como Twitter, Facebook, Periscope o YouTube, por tanto, siguieron el modelo tradicional de emisión previo a la web 2.0.

\section{RTVE siguiendo las directrices del} Mandato-marco (2007) a la Corporación RTVE, manifiesta un claro compromiso de servicio público facilitando la cesión de la señal a múltiples medios que lo solicitaron y abriendo nuevas ventanas de distribución de la señal en directo en plataformas como Twitter, Facebook, Periscope, YouTube, la web corporativa o las apps 
En segundo lugar, se comprueba que los debates electorales siguen concentrando un alto número de espectadores ya que fueron líderes en su franja horaria, así como las emisiones más vistas del mes de abril. De hecho, el debate emitido por TVE fue el espacio no deportivo más visto de todo el año, mientras que el retransmitido por Antena 3 y La sexta ocupó el cuarto y décimo cuarto puesto respectivamente. No obstante, se percibe un progresivo descenso en el índice de audiencias de cada una de estas citas a lo largo de los años y un incremento en la hiperfragmentación de las audiencias. El contexto televisivo de 1993 o 2008 es muy diferente al actual tras el apagón analógico y la explosión de las OTT. El número de emisores se ha multiplicado, por lo que cada vez es más complicado alcanzar altos índices de audiencia. No obstante, estos datos demuestran que la retransmisión de acontecimientos en directo puede servir de ayuda a las cadenas generalistas en su lucha con los nuevos emisores.

En tercer lugar, en lo que se refiere a la producción de mensajes, se comprueba que hubo un importante engagement, así como que Twitter se impuso entre las redes sociales en abierto a la hora de comentar estos espacios televisivos. Ambas retransmisiones fueron trendig topic y además lideraron los índices de audiencia social. Resulta de interés señalar la escasa participación de usuarios anónimos en los tweets examinados y la alta presencia de mensajes de medios de comunicación y partidos políticos, por lo que la participación ciudadana es baja frente a los emisores tradicionales. También, destacan como tweets más retweeteados y favoritos los que incluyen frases en clave espectacular o cuestiones anecdóticas (infotainment).

Tras Twitter, llama la atención la alta producción que se creó en Facebook durante la emisión del espacio de TVE. A pesar de que el número de seguidores del espacio en directo no fue muy elevado, la proporción espectadores en la red/ comentarios fue muy alta, superior a la que se produjo en YouTube.

Finalmente, se puede concluir que la producción de mensajes se realiza principalmente en las redes sociales creadas originariamente para ello, mientras que es más difícil conseguirlo en otras plataformas como YouTube o Periscope. Como indica Andò (2018), estos actos de engagement producen interpretaciones de textos mediáticos y alimentan la circulación de significados además de extender la vida de estos contenidos televisivos. De ahí que constituyan acciones muy relevantes en un ambiente de televisión líquida como el actual.

Esta investigación inaugura nuevas líneas de trabajo sobre el estudio del contenido de los comentarios versados en cada una de las plataformas y las posibles diferencias entre ambas. Asimismo, resultaría de interés comparar la emisión de los debates con otros acontecimientos transmitidos en directo, así como incorporar a los análisis otras redes sociales como Instagram.

\section{Notas}

1. A excepción del celebrado en 2015 en Antena 3 y La sexta al que acudió la vicepresidenta Soraya Sáenz de Santamaría en representación del candidato Mariano Rajoy.

2. Según los datos de Barlovento (2019), el número de tweets estaría muy igualado: el debate de Atresmedia produjo 2.732.878 tweets y el de RTVE 2.720.203.

\section{Referencias}

Andò, Romana (2018). "Esperienze televisive. Schermi, rituali e pratiche delle audience connesse”. En: Andò, Romana; Marinelli, Alberto. Television(s). Come cambia l'esperienza televisiva tra tecnologie convergenti e pratiche social. Milan: Guerini e Associati, pp. 161-198. ISBN: 9788881074150

Apablaza-Campos, Alexis (2017). "Periscope y la videorealidad periodística". En: I Congreso internacional de periodismo: Convergencias mediáticas y nueva narrativa latinoamericana, 5-7 mayo, pp. 141-153. ISBN: 9789978551608 http://ciespal.org/wp-content/uploads/2015/10/ACTAS-Periodismo-FINAL.pdf

Apablaza-Campos, Alexis (2018a). "Social media live streaming (SMLS) in the digital news media". Comunicació: revista de recerca i d'anàlisi, v. 35, n. 2, pp. 103-123.

https://www.raco.cat/index.php/Comunicacio/article/view/355932/447895

Apablaza-Campos, Alexis (2018b). "Social media live streaming y su impacto en los medios de comunicación: El caso de YouTube Live". Hipertext.net, n. 17, pp. 118-128.

https://www.raco.cat/index.php/Hipertext/article/view/10.31009-hipertext.net.2018.i17.11/Apablaza-Campos

Apablaza-Campos, Alexis; Codina, Lluís (2018). "Social media live streaming: estudio de caso y diseño de matriz de análisis". Cuadernos.info, n. 43, pp. 161-180.

https://doi.org/10.7764/cdi.43.1342 
Ballesteros-Herencia, Carlos A. (2019). "La representación digital del engagement: hacia una percepción del compromiso a través de acciones simbólicas”. Revista de comunicación, v. 18, n. 1, pp. 215-233.

https://revistadecomunicacion.com/article/view/1033

Barlovento (2019). Análisis mensual del comportamiento de la audiencia TV. Abril, 2019.

https://www.barloventocomunicacion.es/audiencias-mensuales/analisis-de-audiencia-tv-abril-2019

Bergillos, Ignacio (2017). "Invitaciones a la participación de la audiencia a través de la televisión en España: análisis del prime time de los canales generalistas en 2010 y 2014". Quaderns del CAC, v. 20, n. 43, pp. 89-102.

https://www.cac.cat/sites/default/files/2019-01/Q43_Bergillos_CA.pdf

Berrocal-Gonzalo, Salomé; Cebrián-Guinovart, Elena (2009). “El 'infoentretenimiento' político televisivo. Un análisis de las primeras intervenciones de Zapatero y Rajoy en Tengo una pregunta para usted". Textual \& visual media, n. 2, pp. 41-60. http://textualvisualmedia.com/index.php/txtvmedia/article/view/27/23

Boyle, Raymond (2019). "The television industry in the multiplatform environment". Media, culture \& society, v. 41, n. 7, pp. 919-922.

https://doi.org/10.1177/0163443719868389

Casado-Ruiz, Antonio (2012). "Debates electorales en televisión: nuevos contenidos para nuevos formatos". En: III Congrés Internacional Associació Espanyola d'Investigació de la Comunicació, pp. 138-157. ISBN: 9788461556786 https://dialnet.unirioja.es/servlet/articulo?codigo=5251610

Cebrián-Herreros, Mariano (2004). La información en televisión: obsesión mercantil y política. Barcelona: Gedisa. ISBN: 8497840771

CIS (2018). Barómetro de mayo 2018. Avance de resultados. Estudio n. 3213. Centro de Investigaciones Sociológicas. http://datos.cis.es/pdf/Es3213mar_A.pdf

Claes, Florencia; Deltell, Luis (2015). "Audiencia social en Twitter: hacia un nuevo modelo de consumo televisivo". Trípodos, n. 36, pp. 111-132.

http://www.tripodos.com/index.php/Facultat_Comunicacio_Blanquerna/article/view/245/205

Claes, Florencia; Deltell, Luis; Congosto, Mari-Luz (2015). “Audiencia social ¿comunidad o enjambre? caso de estudio: Goyas 2014". Ar@cne: Revista electrónica de recursos en internet sobre geografía y ciencias sociales, n. 194, pp. 1-15. http://www.ub.edu/geocrit/aracne/aracne-194.pdf

Congosto, Mari-Luz; Deltell, Luis; Claes, Florencia; Osteso, José-Miguel (2013). "Análisis de la audiencia social por medio de Twitter. Caso de estudio: Ios premios Goya 2013". Icono 14, v. 11, n. 2, pp. 53-82.

https://doi.org/10.7195/ri14.v11i2.577

De-Aguilera-Moyano, Miguel; Castro-Higueras, Antonio; Pérez-Rufí, José-Patricio (2018). "Between broadcast yourself and broadcast whatever: YouTube's homepage as a synthesis of its business strategy". El profesional de la información, v. 28, n. 2, pp. 280-206.

https://doi.org/10.3145/epi.2019.mar.06

Deltell, Luis; Claes, Florencia; Osteso, José-Miguel (2013). “Audiencias televisivas y líderes de opinión en Twitter. Caso de estudio: El Barco". Estudios sobre el mensaje periodístico, v. 19, n. 1, pp. 347-364. https://doi.org/10.5209/rev_ESMP.2013.v19.n1.42526

D'heer, Evelien; Verdegem, Pieter (2015). "What social media data mean for audience studies: A multidimensional investigation of Twitter use during a current affairs TV programme". Information, communication \& society, v. 18, n. 2, pp. $221-234$. https://doi.org/10.1080/1369118X.2014.952318

Elmer, Greg (2013). "Live research: Twittering an election debate”. New media \& society, v. 15, n. 1, pp. 18-30. https://doi.org/10.1177/1461444812457328

España (2007). “Ley 17/2006, de 5 de junio, de aprobación por los Plenos del Congreso de los Diputados y del Senado el mandato-marco a la Corporación RTVE previsto en el artículo 4 de la Ley 17/2006, de 5 de junio, de la radio y la televisión de titularidad estatal". BOE, n. 470, 18 diciembre.

http://www.RTVE.es/contenidos/documentos/MANDATO_MARCO_18_12_07.pdf

Gallardo-Camacho, Jorge; Lavín, Eva; Fernández-García, Paula (2016). “Los programas de televisión deportivos y su relación con la audiencia social en Twitter en España”. Revista latina de comunicación social, n. 71, pp. 272-286.

http://www.revistalatinacs.org/071/paper/1095/15es.html

Gallego-Reguera, María; Bernárdez-Rodal; Asunción (2018). “Influencia y repercusión mediática de los debates 'cara a cara' celebrados ante las elecciones generales de 2008 en España: José Luis Rodríguez Zapatero (PSOE) vs Mariano Rajoy (PP)". Vivat academia, n. 141.

https://doi.org/10.15178/va.2017.141.139-154 
García-Avilés, José-Alberto (2011). “Dimensiones y tipología de las actividades de participación de la audiencia en la televisión pública". Ámbitos, n. 20, pp. 175-194.

https://idus.us.es/handle/11441/71549

García-Ferrando, José (2014). “Análisis de la correlación entre audiencias sociales y audiencias televisivas. El caso de Masterchef 2". Cuadernos de gestión de información, v. 4, pp. 213-223.

https://revistas.um.es/gesinfo/article/view/222141

García-García, Alberto; Vinader-Segura, Raquel; Abuín-Vences, Natalia (2012). "Nuevas fórmulas de producción audiovisual atendiendo a criterios de interactividad". En: León-Anguiano, Bienvenido (coord.). La televisión ante el desafío de internet. Salamanca: Comunicación Social, pp. 122-129. ISBN: 9788415544 14-2

Gómez-Aguilar, Marisol; Paniagua-Rojano, Francisco-Javier; Farias-Batlle, Pedro (2015). “Comportamiento de la audiencia de televisión en las redes sociales. Una aproximación al perfil y programas más comentados". Revista latina de comunicación social, n. 70, pp. 539-551.

https://doi.org/10.4185/RLCS-2015-1058

Gómez-Domínguez, Pablo (2019). “La televisión social: reflexiones, recorrido del fenómeno y datos en España”. Obervatori de la producció audiovisual, 12 septiembre.

https://www.upf.edu/web/opa/inici/-/asset_publisher/NSG/4p/BBHKh/content/id/228407371

Gómez-Rubio, Leire; López-Vidales, Nereida (2015). “Del éxito en televisión a la participación en las redes sociales. El Príncipe y Galerías Velvet en Facebook". Doxa.comunicación, n. 20, pp. 137-160.

https://repositorioinstitucional.ceu.es/bitstream/10637/7296/1/Exito_LeireGomez\%26NereidaLopez_Doxa_2015.pdf

González-Neira, Ana; Quintas-Froufe, Natalia (2014). “Audiencia tradicional frente a audiencia social: un análisis comparativo en el prime-time televisivo". Revista mediterránea de comunicación, v. 5, n. 1, pp. 105-121.

https://doi.org/10.14198/MEDCOM2014.5.1.02

González-Neira, Ana; Quintas-Froufe, Natalia (2015). "Revisión del concepto de televisión social y sus audiencias". En: Quintas-Froufe, Natalia; González-Neira, Ana. La participación de la audiencia en la televisión: de la audiencia activa a la social. Madrid: AIMC, pp. 13-26. ISBN: 9788460842422

https://ruc.udc.es/dspace/handle/2183/15956

Halpern, Daniel; Quintas-Froufe, Natalia; Fernández-Medina, Francisco (2016). "Interacciones entre la televisión y su audiencia social: hacia una conceptualización comunicacional”. El profesional de la información, v. 25, n. 3, pp. 367-375. https://doi.org/10.3145/epi.2016.may.06

Herranz-Rubio, Carolina (2020). “La producción científica sobre debates electorales en España: Análisis bibliométrico (1993-2018)". Revista mediterránea de comunicación, v. 11, n. 1, pp. 89-106.

https://www.doi.org/10.14198/MEDCOM2020.11.1.2

Herrero-de-la-Fuente, Mercedes (2017). “Nuevas fórmulas para la televisión en directo: el uso de Facebook Live en Atresmedia". Miguel Hernández communication journal, n. 8, pp. 521-563.

https://doi.org/10.21134/mhcj.v0i8.202

Herrero-de-la-Fuente, Mercedes; García-Domínguez, Antonio (2019). "Facebook Live y la televisión social: el uso del streaming en Antena 3 y laSexta". Vivat academia, v. 146, pp. 43-70.

https://doi.org/10.15178/va.2019.146.43-70

Highfield, Tim; Harrington, Stephen; Bruns, Axel (2013). "Twitter as a technology for audiencing and fandom: The \#Eurovision phenomenon". Information, communication \& society, v. 16, n. 3, pp. 315-339.

https://doi.org/10.1080/1369118X.2012.756053

Humanes, María-Luisa (2014). “Exposición selectiva y partidismo de las audiencias en España. El consumo de información política durante las campañas electorales de 2008 y 2011”. Palabra clave, v. 17, n. 3, pp. 773-802.

https://doi.org/10.5294/pacla.2014.17.3.9

IAB (2019). Estudio anual de redes sociales 2019.

https://iabspain.es/wp-content/uploads/estudio-anual-redes-sociales-iab-spain-2019_vreducida.pdf

Jenkins, Henry; Ito, Mizuko; Boyd, Danah (2015). Participatory culture in a networked era: A conversation on youth, learning, commerce, and politics. Cambridge: Polity Press. ISBN: 9781509503230

Kalsnes, Bente; Krumsvik, Arne H.; Storsul, Tanja (2014). "Social media as a political backchannel: Twitter use during televised election debates in Norway". Aslib journal of information management, v. 66, n. 3, pp. 313-328.

https://doi.org/10.1108/AJIM-09-2013-0093

Larsson, Anders-Olof (2013). "Tweeting the viewer: Use of Twitter in a talk show context". Journal of broadcasting \& electronic media, v. 57, n. 2, pp. 135-152. 
Lee, Jiyoung; Choi, Yun-Jun (2018). "Understanding social viewing through discussion network and emotion: A focus on South Korean presidential debates". Telematics and informatics, v. 35, n. 5, pp. 1382-1391.

https://doi.org/10.1016/j.tele.2018.03.009

Livingstone, Sonia (2004). "The challenge of changing audiences: Or, what is the audience researcher to do in the age of the internet?". European journal of communication, v. 19, n. 1, pp. 75-86.

https://doi.org/10.1177/0267323104040695

Mancebo-García, Marina (2016). "Innovación en formatos audiovisuales. El uso de herramientas de live streaming en Antena 3 y Univision". Miguel Hernández communication journal, n. 7, pp. 325-349.

https://doi.org/10.21134/mhcj.v0i7.152

Marín, Benjamín (2003). “Debates electorales por televisión”. En: Berrocal-Gonzalo, Salomé. Comunicación política en televisión y nuevos medios. Barcelona: Ariel, pp. 207-244. ISBN: 8434412969

Márquez-Martínez, Lucía (2017). “¿Son los debates electorales la nueva Eurovisión? Análisis del seguimiento de dos eventos masivos a través de Twitter". Dígitos, v. 1, n. 3, pp. 137-162.

https://revistadigitos.com/index.php/digitos/article/view/96

Marta-Lazo, Carmen; Mancho-Laiglesia, Ana; Villa-Navarro, Javier (2017). "Cuarta pantalla en los medios de comunicación: análisis de Periscope". Trípodos, n. 40, pp. 91-112.

http://www.tripodos.com/index.php/Facultat_Comunicacio_Blanquerna/article/view/451/598

Martín, Virginia; Vázquez, Tamara; Cebrián, Elena (2017). “Los formatos del politainment televisivo”. En: Berrocal-Gonzalo, Salomé. Politainment. La política espectáculo en los medios de comunicación. Valencia: Tirant Humanidades, pp. 53-76. ISBN: 9788416786206

Ortells-Badenes, Sara (2009). "La mercantilización de la información: la nueva era informativa en televisión". Revista latina de comunicación social, n. 64, pp. 341-353.

http://www.revistalatinacs.org/09/art/28_827_46_ULEPICC_07/Sara_Ortells.html

Pallarés-Navarro, Sandra; García-Ortega, Carmela (2017). “Análisis comparativo del discurso de Mariano Rajoy en Twitter durante las dos campañas electorales de 2015: las autonómicas del 24M y las generales del 20D”. Dígitos, v. 1, n. 3, pp. 119-136.

https://revistadigitos.com/index.php/digitos/article/view/82

Papacharissi, Zizi A. (2015). Affective publics. Sentiment, technology, and politics. New York: Oxford University Press, ISBN: 9780199999736

Quintas-Froufe, Natalia; González-Neira, Ana (2016). “Consumo televisivo y su medición en España: camino hacia las audiencias híbridas". El profesional de la información, v. 25, n. 3, pp. 376-383.

https://doi.org/10.3145/epi.2016.may.07

Quintas-Froufe, Natalia; González-Neira, Ana; Díaz-González, María-Jesús (2015). "La construcción de la estrategia comunicativa en Twitter de un falso documental: Operación Palace”. Revista latina de comunicación social, n. 70, pp. $28-48$. http://www.revistalatinacs.org/070/paper/1033-UC/03es.html

Raman, Aravindh; Tyson, Gareth; Sastry, Nishanth (2018). "Facebook (a)live?: Are live social broadcasts really broadcasts?". En: Proceedings of the 2018 world wide web conference, pp. 1491-1500.

https://arxiv.org/pdf/1803.02791.pdf

Rímoli, Roberto (ed.) (2018). Compendio experimental sobre comunicación política electoral y sus vínculos con la psicología. Miami: SV Graphiccon. ISBN: 9781617901270

Rodríguez-Mateos, David; Hernández-Pérez, Tony (2015). "Televisión social en series de ficción y nuevos roles del documentalista audiovisual: el caso de El ministerio del tiempo". Index comunicación, v. 5, n. 3, pp. 95-120.

http://journals.sfu.ca/indexcomunicacion/index.php/indexcomunicacion/article/view/200/177

Saavedra-Llamas, Marta; Rodríguez-Fernández, Leticia (coords.) (2016). Audiencia social en España: Estrategias de comunicación para medios y marcas. Madrid: Síntesis. ISBN: 9788490774397

Saavedra-Llamas, Marta; Rodríguez-Fernández, Leticia (2018). “Las cadenas de televisión españolas frente al debate del 13J: estrategias de programación y audiencia social”. Fonseca, journal of communication, n. 17, pp. 125-136.

https://doi.org/10.14201/fjc201817125136

Van-Dijck, José (2013). The culture of connectivity: A critical history of social media. New York: Oxford University Press, ISBN: 9780199970773

Van-Holsteyn, Joop J. M. (2018). "The Dutch parliamentary elections of March 2017". West European politics, v. 41, n. 6, pp. 1364-1377.

https://doi.org/10.1080/01402382.2018.1448556 